\title{
Conversión de la prueba de tuberculina en un programa de residencia en pediatría en México: estudio prospectivo de cinco años
}

\author{
Tuberculin test conversion in a pediatric residency program \\ in Mexico: a five-year prospective study
}

\author{
Abiel Mascareñas de los Santos,* Denisse Vaquera Aparicio, ${ }^{*}$ Juan Carlos Luévanos Gurrola, ${ }^{*}$ \\ Carlos Alberto Hernández Salas, ${ }^{\ddagger}$ Fernando García Rodríguez, ${ }^{\ddagger}$ \\ Manuel de la O-Cavazos, ${ }^{\ddagger}$ José Iván Castillo Bejarano* \\ * Departamento de Pediatría, Servicio de Enfermedades Infecciosas. \\ ₹ Departamento de Pediatría.
}

Hospital Universitario «Dr. José Eleuterio González». Universidad Autónoma de Nuevo León. Monterrey, México.

\section{RESUMEN}

Introducción: En México, el tamizaje para detección de tuberculosis latente no es un programa sistematizado. El objetivo fue evaluar la prevalencia, tasa de incidencia y factores de riesgo para la conversión del péptido purificado derivado (PPD) en un programa de residencia en pediatría en Nuevo León, México. Material y métodos: Estudio de cohorte, involucró residentes de un hospital público en México, se recolectaron datos demográficos y clínicos. Una induración de PPD $\geq 10 \mathrm{~mm}$ fue considerada positiva. La conversión de PPD se definió como un resultado $\geq 10 \mathrm{~mm}$ en estudiantes con un resultado negativo previo. Se utilizó la prueba de rangos de Wilcoxon y se realizó un análisis logístico de regresión para medir la asociación de posibles factores para la reactividad del PPD. La tasa de conversión fue sometida a la prueba de McNemar. Resultados: Noventa y nueve estudiantes fueron seguidos, $54 \%$ fueron mujeres. La prevalencia basal de PPD positivo fue $21 \%$. La prevalencia de la conversión fue $25 \%$. La conversión fue mayor (20.4\%) en residentes del primer año. La tasa de conversión fue de 10.5/1,000 personas-meses, con una mayor prevalencia en residentes de primer año (13.6/1,000 personas-meses). La probabilidad de presentar un resultado positivo basal fue más alta en estudiantes con exposición previa a un caso de tuberculosis (OR; 3.36 [95\% IC 1.3-8.2]). Conclusiones: Nuestro estudio identifica una alta prevalencia e incidencia de conversión de PPD en residentes de pediatría en México. El diseño de estrategias preventivas más estrictas es necesario para prevenir la infección por tuberculosis en nuestra población.

Palabras clave: Tuberculosis, prueba cutánea de tuberculina, tuberculosis latente, Mantoux.

\section{ABSTRACT}

Introduction: In Mexico, screening for latent tuberculosis is not a systematized program. The objective was to evaluate the prevalence, incidence rate and risk factors for purified peptide derivative (PPD) conversion in a pediatric residency program in Nuevo Leon, Mexico. The objective was to evaluate the prevalence, incidence rate and risk factors for purified peptide derivative (PPD) conversion in a pediatric residency program in Nuevo Leon, Mexico. Material and methods: Cohort study, involving residents of a public hospital in Mexico, demographic and clinical data were collected. A PPD induration $\geq 10 \mathrm{~mm}$ was considered positive. PPD conversion was defined as a result $\geq 10 \mathrm{~mm}$ in students with a previous negative result. The Wilcoxon rank test was used and a logistic regression analysis was performed to measure the association of possible factors for PPD reactivity. The conversion rate was subjected to McNemar's test. Results: 99 students were followed up, $54 \%$ were female. The baseline prevalence of positive PPD was $21 \%$. The prevalence of conversion was $25 \%$. Conversion was higher (20.4\%) in first-year residents. The conversion rate was 10.5/1,000 person-months, with a higher prevalence in first-year residents (13.6/1,000 person-months). The probability of presenting a baseline positive result was higher in students with previous exposure to a case of tuberculosis (OR; 3.36 [95\% Cl 1.3-8.2]). Conclusions: Our study identifies a high prevalence and incidence of PPD conversion in pediatric residents in Mexico. The design of more stringent preventive strategies is necessary to prevent tuberculosis infection in our population.

Keywords: Tuberculosis, tuberculin skin test, latent tuberculosis, Mantoux test.

\footnotetext{
Citar como: Mascareñas SA, Vaquera AD, Luévanos GJC, Hernández SCA, García RF, De la O-Cavazos M et al. Conversión de la prueba de tuberculina en un programa de residencia en pediatría en México: estudio prospectivo de cinco años. Rev Latin Infect Pediatr. 2021; 34 (4): 179-182. https://dx.doi.org/10.35366/102967
}

Recibido: 03-09-2021. Aceptado: 08-10-2021.

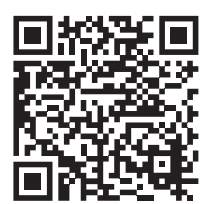




\section{INTRODUCCIÓN}

Los trabajadores de la salud (TS) representan un grupo de alto riesgo para desarrollar tuberculosis latente (TL). ${ }^{1}$ Los hospitales de países en desarrollo cuentan con programas de vigilancia para monitorizar la TL en los trabajadores de la salud; ${ }^{2,3} \sin$ embargo, en México esta vigilancia no se lleva a cabo de forma sistemática.

Los programas de residencias constituyen un área de oportunidad donde los protocolos de vigilancia hospitalaria no han sido definidos adecuadamente y se cuenta con información escasa sobre la prevalencia de conversión en la prueba cutánea de derivado proteico purificado (PPD). ${ }^{4}$ Nuestro objetivo fue identificar la prevalencia y tasa de incidencia de PPD positiva y la conversión en residentes de pediatría en un hospital universitario en México.

\section{MATERIAL Y MÉTODOS}

Este estudio de cohorte se realizó en el Hospital Universitario «Dr. José Eleuterio González» en el noreste de México del año 2016 a 2020. En seguimiento al surgimiento de dos casos de tuberculosis (TB) en residentes del Departamento de Pediatría en 2016, se estableció un plan para la aplicación de PPD en dos tiempos. Se recolectaron características demográficas, contacto con casos confirmados de tuberculosis, estatus de vacunación con bacilo de Calmette-Guérin (BCG), y síntomas pulmonares de tuberculosis.

Utilizando el método de Mantoux, dos unidades de tuberculina $(0.1 \mathrm{~mL})$ de derivado purificado proteínico (PPD) fueron administradas de manera intradérmica con una lectura a las 72 horas. Una prueba positiva fue definida como una induración de la piel $\geq 10 \mathrm{~mm}$. Una conversión de una PPD fue definida como un cambio $\geq 10 \mathrm{~mm}$ posterior a un resultado negativo previo. Todas las pruebas fueron aplicadas y analizadas por el mismo médico. En residentes de pediatría con resultados de base positivos y en los que presentaron conversión de PPD, se realizó una radiografía de tórax. Se contó con la aprobación del Comité de Ética Institucional Universitaria previo al inicio del estudio y al reclutamiento de los participantes.

Los datos fueron analizados utilizando el programa SPSS V.21 (SPSS Inc., Chicago, IL, USA). Las variables continuas fueron expresadas como medianas e intervalos intercuartiles (IQI). Las variables categóricas fueron expresadas como frecuencias y porcentajes. Se calcularon los intervalos de confianza a 95\% y la razón de probabilidades (OR). Los datos de la tasa de conversión fueron sometidos a una prueba de análisis de McNemar para medir la diferencias en las tasas de conversión. La prueba de los rangos de Wilcoxon fue utilizada como comparación de las variables continuas. Para los residentes con resultados negativos de base, se calculó la tasa de conversión de PPD por 1,000 personas-meses. Un valor de $p \leq 0.05$ fue considerado estadísticamente significativo.

\section{RESULTADOS}

De 2016 a 2020, un total de 99 estudiantes de postgrado fueron enrolados después de dar consentimiento (Figura 1), 54\% fueron mujeres, la edad media del grupo fue de 27 años. El antecedente de vacunación con BCG fue documentado en $93 \%$ de los residentes. Ninguno de los participantes presentó comorbilidades durante el periodo de estudio.

De los 99 estudiantes, 21 (21\%) presentaron un resultado basal positivo con una induración media de $13 \mathrm{~mm}$ (rango de 12-17 mm). La mediana de duración en el programa de postgrado durante la primera prueba fue de 11 meses (rango de 3-23 meses) (Figura 2). Un resultado positivo fue más probable en residentes con vacuna $B C G(O R ; 1.29$ [95\% IC 0.2-8.0]) y un contacto previo con un caso de tuberculosis (OR; 3.36 [95\% IC 1.3-8.2]).

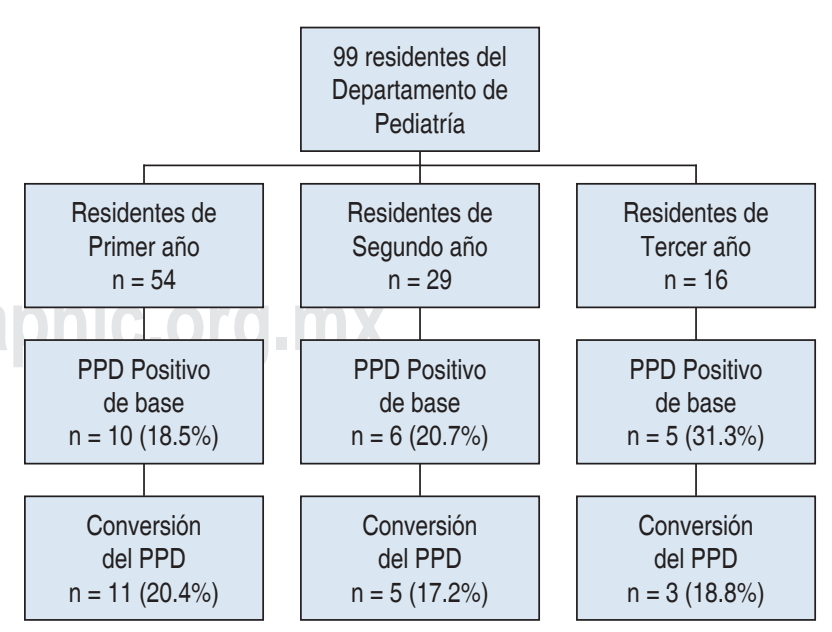

Figura 1: Resultados de péptido purificado derivado (PPD) en residentes del Departamento de Pediatría 2016 a 2020. 
A

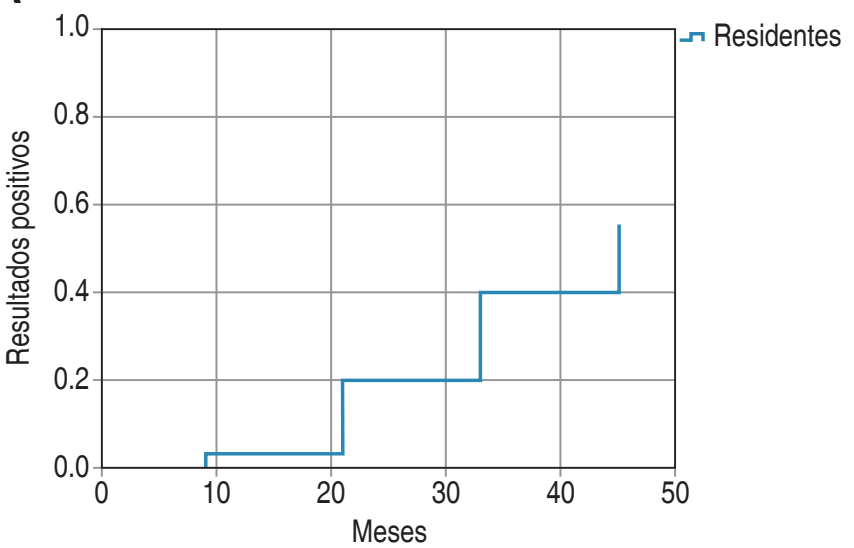

B

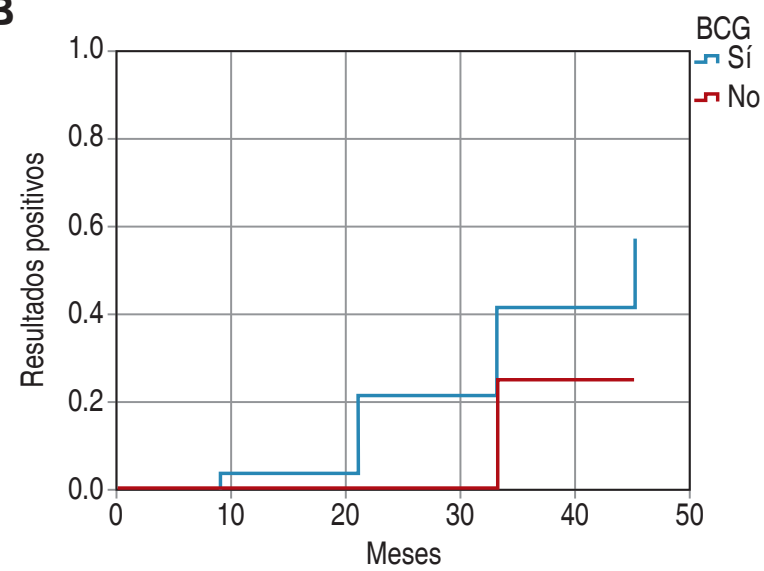

Figura 2: A) Proporción acumulada de residentes con resultado de péptido purificado derivado (PPD) positivo a través del tiempo. B) Proporción acumulada de acuerdo con el estatus de vacunación con bacilo de Calmette-Guérin (BCG).

Entre los 78 residentes con un resultado negativo basal, 76 (97\%) tuvieron una prueba de seguimiento. La conversión de PPD ocurrió en 19 (25\%) residentes con una induración media de 14 mm (rango 10-15). Aunque la prevalencia de conversión fue más alta para los residentes de primer año (20.4\%), no se encontró diferencia estadística entre los grupos $(p$ $>0.05$ ). La prevalencia global de reactividad en el PPD fue de $40 \%$. Un contacto reciente con un caso de TB no fue documentado durante la aplicación de la segunda prueba.

La tasa de conversión de PPD fue de 10.5 por 1,000 personas-meses. La tasa de conversión de PPD fue más alta en residentes de primer año (13.6/1,000 personas-meses) comparada con la conversión de los residentes de segundo año (10.4/1,000 personas-meses). El tiempo medio de duración en el programa de residencias para la conversión de la prueba fue de 24 meses (rango 14-32 meses). Como un factor de riesgo independiente, la vacuna con BCG (OR; 0.8 [95\% IC 0.7-0.9]) y la cicatriz de la BCG no fueron significantes para la conversión de la PPD (OR; 0.9 [95\% IC 0.2-2.8]). EI análisis para evaluar la diferencia en la proporción de residentes con PPD positiva fue estadísticamente significativa $(p<0.05)$ (Tabla 1).

En cuanto a los síntomas pulmonares sugestivos de tuberculosis, sólo ocho (8\%) residentes describieron presentar tos por más de 15 días como único síntoma. Sólo un residente desarrolló TB pulmonar durante el estudio. De acuerdo con las radiografías de tórax, ningún residente presentó anormalidades.

\begin{tabular}{|c|c|}
\hline Características & OR (IC 95\%) \\
\hline \multicolumn{2}{|l|}{ PCT de Base } \\
\hline \multicolumn{2}{|l|}{ Género } \\
\hline \multicolumn{2}{|l|}{ Año de administración de la PCT } \\
\hline $\begin{array}{l}\quad<2018 \\
\text { Generación de residentes }\end{array}$ & $0.89(0.38-2.05)$ \\
\hline 2014-16 & $1.2(0.57-2.63)$ \\
\hline 2017-19 & $0.68(0.27-1.70)$ \\
\hline $2020-21$ & $1.1(0.39-3.39)$ \\
\hline Vacunación con BCG & $1.2(0.20-8.05)$ \\
\hline Cicatriz de BCG & $0.1(0.25-1.13)$ \\
\hline $\begin{array}{l}\text { Contracto previo con caso infeccioso de TB } \\
\text { Sí }\end{array}$ & $3.36(1.3-8.25)$ \\
\hline \multicolumn{2}{|l|}{ Conversión de la PCT } \\
\hline \multicolumn{2}{|l|}{ Género } \\
\hline Masculino & $0.62(0.25-1.5)$ \\
\hline \multicolumn{2}{|l|}{ Año de administración de la PCT } \\
\hline$<2018$ & $1.3(0.49-3.9)$ \\
\hline \multicolumn{2}{|l|}{ Generación de residencia } \\
\hline $2014-16$ & $1.4(0.61-3.24)$ \\
\hline 2017-19 & $0.89(0.36-2.20)$ \\
\hline $2020-21$ & $0.72(0.25-2.04)$ \\
\hline Vacunación de la BCG & $0.85(0.79-0.91)$ \\
\hline Cicatriz de la BCG & $0.9(0.29-2.8)$ \\
\hline
\end{tabular}




\section{DISCUSIÓN}

Encontramos una alta prevalencia de PPD positivo $(40 \%)$ en residentes de pediatría en México, con una conversión de la PPD de $25 \%$. La posibilidad de PPD positivo basal fue más probable en residentes con vacuna BCG y con exposición documentada a un caso de TB. Las limitaciones de nuestro estudio fueron la falta de IGRA (QuantiFERON ${ }^{\mathrm{TM}}$-TB Gold) y una población más grande para analizar la información.

La información en cuanto a la prevalencia de una prueba de PPD reactiva en México es escasa, Ostrosky-Zeichner y colaboradores ${ }^{5}$ describieron una prevalencia de positividad basal en la PPD de 39.6\% con un $43.3 \%$ de conversión entre TS en la Ciudad de México. En nuestra investigación, encontramos una prevalencia de reactividad a la PPD de $40 \%$, comparable con el reporte nacional previo, a pesar de haber transcurrido 20 años entre ambos estudios. En otros países con prevalencia intermedia y baja de TB, se describe una positividad de la PPD de 46 y $2.5 \%$. ${ }^{6,7}$

Una revisión sistémica previa describió una prevalencia de TL de $2 \%$ en estudiantes de medicina en Irán, que correlaciona con la prevalencia de la población general del país. ${ }^{8}$ Chung-Delgado $\mathrm{K}$ y su grupo ${ }^{9}$ reportaron en estudiantes de medicina una prevalencia de PPD reactivo de $14.4 \%$, en comparación con 19\% de positividad del PPD de nuestro estudio. En países con alta carga de TB, se reportan tasas de conversión de PPD de 10.7 por 1,000 personas-meses entre TS, similar a 10.5 por 1,000 personas-meses de nuestro estudio. ${ }^{10}$

De acuerdo con la tasa de conversión de PPD entre residentes, observamos una alta tasa entre estudiantes de primer año de residencia. Los casos de TB en niños son considerados paucibacilares, así que la incidencia de PPD positivo puede estar asociada con otros factores. En comparación con un reporte de Irán, la conversión de la PPD en residentes puede estar asociada con la prevalencia de casos de TB en México.

\section{CONCLUSIONES}

Nuestro estudio identifica una alta prevalencia y tasa de incidencia de PPD positivo en un programa de residencia de pediatría en México, comparable con la alta carga de la enfermedad por TB en nuestro país. Considerando estos hallazgos, un programa preventivo debe ser instaurado en los programas de especialización médica en México.

\section{REFERENCIAS}

1. Kim SY, Park MS, Kim YS, Kim SK, Chang J, Kang YA Conversion rates of an interferon- $\gamma$ release assay and the tuberculin skin test in the serial monitoring of healthcare workers. Infection. 2013; 41 (2): 511-516.

2. Edet A, Turner S. Factors associated with positive Mantoux results in NHS healthcare workers. Occup Med (Chic III). 2018; 68 (4): 286-289.

3. Cook S, Maw KL, Munsiff SS, Paula I, Frieden TR. Prevalence of tuberculine skin test positivity and conversions among healthcare workers in New York City during 1994 to 2001. Infect Control Hosp Epidemiol. 2003; 24 (11): 807 813.

4. Mascareñas $\mathrm{SAH}$, Castillo BJI, de la O CME, Rodríguez BI, Treviño GC, Villarreal GRV et al. Perfil clínico y epidemiológico del PPD en residentes de pediatría de un hospital universitario del noreste de México. Rev Latin Infect Pediatr. 2017; 30 (3): 97-101.

5. Ostrosky-Zeichner L, Rangel-Frausto MS, García-Romero E, Vázquez A, Ibarra MJ, Ponce de León-Rosales S. Tuberculosis en trabajadores de la salud: Importancia de los programas de vigilancia y control. Salud Publica Mex. 2000; $42(1): 48-52$.

6. Munisamy M, Krishnan K, Selvaratnam G, Panza A, Pongpanich S, Jimba M. Not Tb-proof: latent tuberculosis in Kuala Lumpur hospital health care workers. Occup Med (Chic III). 2017; 67 (3): 224-226.

7. Joshi R, Reingold AL, Menzies D, Pai M. Tuberculosis among health-care workers in low- and middle-income countries: A systematic review. PLoS Med. 2006; 3 (12): 2376-2391.

8. Golchin M, Mostafa R. Tuberculin test in nursing and humansciences students. J Res Med Sci. 2005; 10 (3): 172-176.

9. Chung-Delgado K, Guillén-Bravo S, Navarro-Huamán L, Quiroz-Portella R, Revilla-Montag A, Ruíz-Alejos A et al. Estudiantes de medicina en riesgo: prevalencia e incidencia de conversión de PPD. Rev Chil Infectol. 2012; 29 (4): 375381.

10. Roth V, Garrett DO, Laserson KF, Starling CE, Kritski AL, Medeiros EAS et al. A multicenter evaluation of tuberculin skin test positivity and conversion among health care workers in Brazilian hospitals. Int J Tuberc Lung Dis. 2005; 9 (12): 1335-1342

Financiamiento: Ninguno.

Conflicto de intereses: Ninguno.

Correspondencia:

José Iván Castillo Bejarano

E-mail: jicastillobejarano@gmail.com 